\title{
Conversión de matrices de dosis en términos de dosis biológica equivalente y dosis equivalente a 2 Gy por sesión
}

\section{Dose matrix conversion in terms of biological effective dose and equivalent dose in 2 Gy fractions}

\author{
Jaime Martí Asenjo ${ }^{1}$, Miguel Ángel de la Casa Julián ${ }^{1}$, Leyre Alonso Iracheta ${ }^{1}$, \\ Alejandro Prado Barragán', Inés Flores Cacho ${ }^{1}$, Beatriz Álvarez Rodríguez², \\ María del Carmen Rubio Rodríguez², Pedro Fernández Letón ${ }^{1}$ \\ 1 Servicio de Radiofísica y Protección Radiológica - Hospital Universitario HM Sanchinarro \\ 2 Servicio de Oncología Radioterápica - Hospital Universitario HM Sanchinarro
}

Fecha de Recepción: 10/11/2020 - Fecha de Aceptación: 16/07/2021

La re-irradiación de pacientes en radioterapia ha sido siempre un proceso complejo. Las herramientas disponibles en los sistemas de planificación de tratamiento actuales permiten generalmente el registro de imágenes y la suma las dosis administradas, pero el uso cada vez más habitual de hipofraccionamientos y esquemas de tratamiento acelerados supone que esta suma de planes sea difícil de evaluar correctamente. Esta circunstancia añade incertidumbre a la definición de volúmenes de tratamiento y la prescripción de dosis. Se ha desarrollado una herramienta para convertir las matrices de dosis de tratamiento en matrices de dosis biológica equivalente y dosis equivalente a 2 Gy por sesión. La herramienta genera archivos de dosis según el estándar DICOM, por lo que pueden ser importados en el sistema de planificación de tratamientos para evaluar la suma de planes con distintos fraccionamientos. El presente estudio describe el proceso de creación de la herramienta y analiza su posible uso clínico. Los resultados muestran una correcta alineación entre las matrices de dosis calculadas y la imagen, así como posibles limitaciones en su uso, especialmente en la evaluación de dosis a punto en los márgenes de las estructuras definidas. El uso de la herramienta es adecuado y útil para la práctica clínica pero requiere una comprensión de su funcionamiento y limitaciones.

Palabras clave: Dosis biológica equivalente, dosis equivalente a 2 Gy por sesión, radiobiología, re-irradiación, dosimetría.

Re-irradiation treatments are complex. Nowadays treatment planning systems have developed tools that allow image registration and add different dose plans on registered images. However, hypofractioned and accelerated treatments remain a challenge as evaluation of summed doses from different fractioning are difficult to understand. On this basis, treatment volume definition and dose prescription are sometimes limited and unclear. A tool has been developed to convert absorbed dose to Biologically Effective Dose and 2 Gy fractions equivalent dose. This tool creates a standard DICOM dose file that is possible to import on to the treatment planning system and allows the evaluation of summed dose plans with different fractioning. This works describes the tool creation and analyzes its clinical use. Results show a correct alignment between calculated dose matrices and the image set. It also shows limitations on the evaluation of point doses, especially in the boundaries of defined structures. Although this tool might be useful in a clinical environment an understanding of its limitations is required.

Key words: BED, EQD2, radiobiology, re-irradiation, dosimetry.

\footnotetext{
*Correspondencia: jmartiasenjo@hmhospitales.com https://doi.org/10.37004/sefm/2021.22.2.001
} 


\section{Introducción}

El número de pacientes que reciben radioterapia como parte de un tratamiento contra el cáncer crece cada año. ${ }^{1}$ La probabilidad de que estos pacientes requieran un nuevo tratamiento tiempo después, ya sea por recidivas del tumor original, metástasis o incluso nuevos tumores es alta. ${ }^{2}$ Esto hace que cada vez con más frecuencia se necesiten segundos, terceros e incluso múltiples tratamientos posteriores. La mayoría de los sistemas de planificación actuales cuentan con herramientas que permiten el registro de imágenes (rígido y deformable) así como la suma de diferentes planes de tratamiento. Esta operación se realiza generalmente como la suma de la dosis absorbida, sin tener en cuenta el tipo de tejido o los fraccionamientos empleados, lo que convierte su correcta evaluación en algo complejo. El uso de esquemas de tratamiento hipofraccionados y acelerados, como en la radioterapia estereotáxica extracraneal (Stereotactic Body Radiation Therapy, SBRT), son habituales y preferibles para el tratamiento de recaídas y metástasis. ${ }^{3-6}$ En ellos se administran altas dosis de radiación en un número pequeño de sesiones, utilizando un fraccionamiento generalmente distinto al empleado para el tratamiento del tumor original. En este contexto, la complejidad de la evaluación de estos tratamientos múltiples se incrementa considerablemente ya que, a fin de evitar toxicidades, es imprescindible tener en cuenta la dosis previa recibida para poder definir los límites dosimétricos en el nuevo tratamiento. El número de artículos relacionados con la re-irradiación de determinadas localizaciones ha crecido considerablemente en los últimos años ${ }^{7-9}$ aunque todavía existe una importante carencia de guías concretas sobre dosis acumuladas y toxicidades. ${ }^{10}$

Es por tanto cada vez más necesario contar con herramientas que permitan la evaluación de los efectos biológicos derivados de la suma múltiples tratamientos con diferentes fraccionamientos, para lo que es necesario la aplicación de modelos de isoefecto. ${ }^{11} \mathrm{La}$ magnitud dosis biológica equivalente (DBE) propuesta por Fowler ${ }^{12}$ (1989) calcula las relaciones de isoefecto basándose en el modelo lineal-cuadrático (LQ) para la supervivencia celular. Este modelo es el más usado para la evaluación radiobiológica en radioterapia para fraccionamientos estándar. ${ }^{13} \mathrm{Ha}$ sido también usado en otros esquemas como en radiocirugía, SBRT, o tratamientos hipofraccionados, ${ }^{14-17}$ aunque su uso genera controversia, tanto para tratamientos de una única sesión ${ }^{18-21}$ como en aquellos casos en que la dosis absorbida por sesión es inferior a 1 Gy.

Además de la DBE, es muy habitual el uso de la denominada dosis equivalente a fraccionamiento estándar de 2 Gy por sesión (DEQ2). Esta magnitud se define como la dosis que habría que suministrar al tejido para conseguir el isoefecto deseado administrando 2 Gy por sesión.

A fin de poder evaluar con seguridad tratamientos complejos, con contribuciones de distintos planes o reirradiaciones de volúmenes, incluso impartidos a distintas dosis por fracción, se ha desarrollado en Matlab un programa para la conversión del archivo exportado de dosis absorbida, obtenida en el sistema de planificación de tratamiento (SPT), a DBE y DEQ2. El archivo convertido puede ser importado de nuevo en el SPT, permitiendo el uso de las herramientas de registro de imágenes y evaluación de tratamientos sobre sumas de planes expresados en DBE o DEQ2.

\section{Material y método}

Los modelos de isoefecto son aquellos que permiten comparar el efecto biológico de la radiación sobre un tejido, independientemente del fraccionamiento empleado. La DBE representa la dosis que habría que suministrar al tejido para conseguir el isoefecto deseado en fracciones infinitamente pequeñas. ${ }^{22}$ La DBE es por tanto aditiva para un determinado efecto en un tejido concreto y el efecto de varias irradiaciones sobre un tejido se puede evaluar como la suma de la DBE de cada una de ellas.

El cálculo de la DBE se ha realizado siguiendo la forma obtenida a partir de la definición del efecto biológico propuesta por Fowler: ${ }^{12}$

$$
E=-\ln (\text { fracción celular superviviente })
$$

De esta manera se establece una relación entre una fracción de células muertas y un isoefecto concreto. Considerando el modelo de supervivencia LQ, que establece el efecto biológico como

$$
E=\alpha n d+\beta n d^{2}
$$

donde $n$ es el número de fracciones, $d$ la dosis por fracción, $\alpha$ y $\beta$ son constantes características de la población celular estudiada. El cociente $\alpha / \beta$ es la dosis en Gy en la que se igualan las contribuciones lineal $(\alpha)$ y cuadrática $(\beta)$, es decir, la dosis a la que la contribución del término lineal iguala a la contribución del término cuadrático. ${ }^{22}$ En este modelo la DBE se define como:

$$
\mathrm{DBE}=\frac{E}{\alpha}=n d\left(1+\frac{d}{\alpha / \beta}\right)
$$

La DEQ2 se obtiene directamente a partir de la ecuación anterior cuando se considera la DBE del tratamiento a 2 Gy por sesión y se igualan los efectos: 


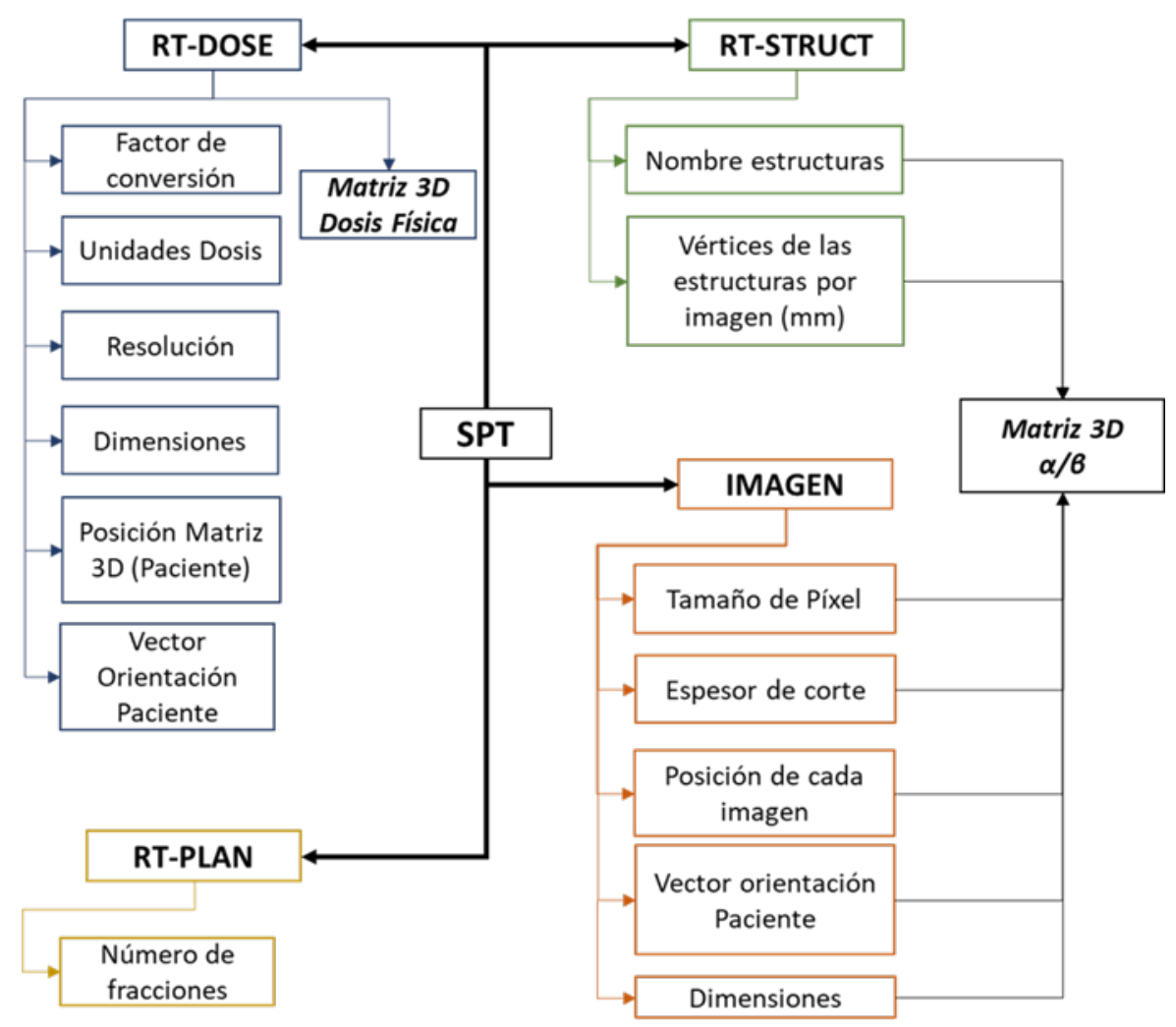

Fig. 1. Esquema de extracción de datos a partir de los archivos exportados del SPT.

$$
\mathrm{DEQ} 2=\frac{\mathrm{DBE}}{\left(1+\frac{2}{\alpha / \beta}\right)}
$$

La DEQ2 es también aditiva al mantener la dosis por fracción y tiene la ventaja de que la mayoría de efectos adversos en tejidos derivados de la radioterapia están descritos para este tipo de fraccionamiento.

El equipamiento empleado para la realización de este trabajo ha sido el SPT Raystation (RaySearch Laboratories, Estocolmo, Suecia) el cual cuenta con un módulo de registro de imágenes con posibilidad de realizar fusiones rígidas y deformables, empleándose como sistema de imagen un equipo de tomografía computarizada (TC) modelo Aquilion LB (Canon Medical System, Otawara, Japón).

Se ha empleado para la edición de las matrices de dosis el software Matlab (C) 1994-2020 The MathWorks, Inc., Natick, Estados Unidos) con el módulo de procesado de imágenes (image processing toolbox). Este módulo es necesario para la edición y generación de archivos DICOM. Mediante las funciones dicominfo y dicomread de Matlab podemos acceder a las matrices numéricas y los metadatos de cada uno de los archivos con extensión dcm (imagen, plan, estructuras y dosis) y extraer la información necesaria para el cálculo. Para la modificación de la matriz de dosis original y la generación del plan de dosis biológica es necesario exportar desde el sistema de planificación las imágenes TC de la simulación, el plan de tratamiento (RT-Plan), el archivo de estructuras (RT-Struct) y la matriz de dosis física (RT-Dose). Todos ellos son archivos estructurados según su estándar DICOM. ${ }^{23}$ De cada uno de ellos se extrae la información necesaria para la construcción del archivo de dosis final, como se muestra en la figura 1.

Para el cálculo de la DBE y la DEQ2 es necesario conocer el número de fracciones administradas en el tratamiento, este dato está en la cabecera de datos del archivo RT plan. Dentro del campo etiquetado como Fraction Group Sequence, se encuentra el Number of Fractions Planned, que contiene el número de fracciones planificadas.

El archivo de estructuras contiene la información necesaria para identificar, sobre el estudio de imagen, los vóxeles que corresponden a cada una de las estructuras contorneadas en el plan de tratamiento. Para cada imagen del estudio, se almacenan las coordenadas de los vértices que delimitan cada una de las estructuras que aparecen en ella. Éstas están almacenadas dentro del campo ROI Contour Sequence de la cabecera del archivo de estructuras, en forma de lista, en milímetros y referidas al sistema de coordenadas de la sala. Para localizar las estructuras en la imagen es necesario convertir las distancias de milímetros a píxeles. Encontramos dentro de cada imagen del estudio, por un 
lado, el tamaño de píxel de la imagen axial en el campo Pixel Spacing y por otro, la posición del vértice superior izquierdo, visto en el sistema de referencia del paciente, en el campo Image Position Patient. La orientación de las imágenes respecto al sistema de referencia de la sala viene descrita en el campo Image Orientation Patient, donde se encuentran los cosenos directores que indican la rotación de un sistema respecto al otro.

La dosis del plan está almacenada en una matriz tridimensional con una profundidad de 16 bits. El vóxel con la dosis máxima del plan se registra con el valor más alto, es decir $2^{16}-1$ (65535), el factor para su conversión a dosis se almacena en el campo Dose Grid Scaling y las unidades en las que está almacenado en el campo Dose Units. Necesitaremos además conocer la resolución de la malla de cálculo, indicada en Pixel Spacing para las dimensiones lateral y vertical, y en Slice Thickness para la dimensión longitudinal, así como el tamaño de la malla: Width para la dimensión lateral y Height para la vertical. La localización exacta de la rejilla de cálculo se encuentra en el campo Image Position Patient. De igual forma que en el archivo de estructuras existe también un campo Image Orientation Patient, donde se almacenan los cosenos directores que nos indican la orientación de la matriz de dosis y del vector Image Position Patient respecto al sistema de coordenadas de la sala.

La construcción de la nueva matriz de dosis se realiza a partir de los datos extraídos de las imágenes TC de la simulación, el plan de tratamiento (RT-Plan), el archivo de estructuras (RT-Struct) y la matriz de dosis física (RT-Dose). Es posible modificar este último para que en lugar de dosis absorbida esté expresado en términos de la DBE o la DEQ2.

Para realizar un cálculo adecuado de la DBE o la DEQ2 es necesario conocer el cociente $\alpha / \beta$ correspondiente a cada vóxel del estudio de imagen. Para ello, creamos primero una matriz tridimensional de ceros, a la que denominamos matriz $\alpha / \beta$, con las mismas dimensiones que el estudio de imagen, normalmente $512 \times 512 \times N$, donde $N$ es el número de cortes. Cada plano $512 \times 512$ de la matriz corresponde por tanto a una imagen concreta. A partir de la información obtenida del archivo RT-Struct conocemos todas las estructuras contenidas en cada una de las imágenes, así como la posición de los vértices de los polígonos que las delimitan. Utilizando el valor del tamaño de píxel, obtenido de la cabecera de la imagen, podemos convertir los vértices del polígono (originalmente en coordenadas de la sala) a valores de filas y columnas que nos permitan identificar los vértices en la matriz $\alpha / \beta$. Así, asignamos a todos los elementos de la matriz contenidos dentro de esta región el valor del cociente $\alpha / \beta$ en función del órgano que haya sido etiquetado y del efecto considerado. Para ello, a partir de valores tomados de la literatura, ${ }^{22,24}$ creamos un diccionario de cocientes $\alpha / \beta$ editable.
Este proceso termina con una matriz tridimensional, que tiene exactamente las mismas dimensiones y por tanto mismo número de vóxeles que el estudio de imagen y donde cada elemento tiene el valor del cociente $\alpha / \beta$ que corresponda a su equivalente en la imagen según el volumen etiquetado en el archivo de estructuras. A todos aquellos elementos que no están contenidos en ningún volumen concreto, o están contenidos en una ROI no presente en el diccionario de cocientes, se les asigna el valor $\alpha / \beta=3$ Gy por considerarse el más habitual. El programa permite editar este valor por defecto. Para los casos en los que un vóxel pertenece a más de una estructura, se establece una jerarquía editable para la asignación del cociente $\alpha / \beta$, por defecto se asigna el de valor inferior.

Tanto el tamaño, como la resolución de la matriz $\alpha / \beta$ y la matriz de dosis no coinciden a priori (fig. 2). La primera viene determinada por el estudio de imagen y la segunda por la malla de cálculo. Para establecer una equivalencia entre cada vóxel de la matriz de dosis y cada uno de la matriz $\alpha / \beta$ es necesario redimensionar una de las dos para que tengan exactamente la misma resolución.

La primera opción es redimensionar la denominada matriz $\alpha / \beta$. Como cada vóxel de dosis va a contener más de un vóxel de imagen, dado que casi siempre es de mayor tamaño, es necesario cambiar la matriz $\alpha / \beta$ disminuyendo su resolución. Por tanto, en general, un vóxel de dosis estará asociado a más de un vóxel de imagen y en consecuencia habrá casos en los que coexistirán valores distintos de $\alpha / \beta$ para un mismo vóxel de dosis, siendo necesario adoptar un único valor. Así, podrían quedar valores de dosis calculados con un determinado $\alpha / \beta$ contenidos en estructuras con un $\alpha / \beta$ distinto, apareciendo efectos de borde significativos entre estructuras con distintos valores de $\alpha / \beta$.

La segunda alternativa es la de redimensionar la matriz de dosis, aumentando su resolución. Esta solución elimina a priori los efectos de borde, pero tiene la limitación de que algunos SPT tienen un tamaño de vóxel mínimo para la malla de dosis a $1 \times 1 \times 1 \mathrm{~mm}$. En la mayoría de los TC de simulación el tamaño de vóxel en los ejes $X$ e $Y$ es inferior a $1 \mathrm{~mm}$, por lo que esta solución sólo es posible en aquellos SPT que admitan, al menos, importar archivos de dosis con tamaños inferiores a $1 \mathrm{~mm}$. El SPT que hemos utilizado (Raystation) no permite calcular a resolución inferior a $1 \mathrm{~mm}$, pero si permite su importación. Es por ello que optamos por redimensionar la matriz de dosis para minimizar los efectos de borde.

Para el redimensionado utilizaremos la función, predefinida en Matlab, griddedInterpolant sobre la matriz de dosis, indicándole el nuevo número de filas, columnas y planos para la nueva matriz. Utilizaremos un interpolado trilineal. 


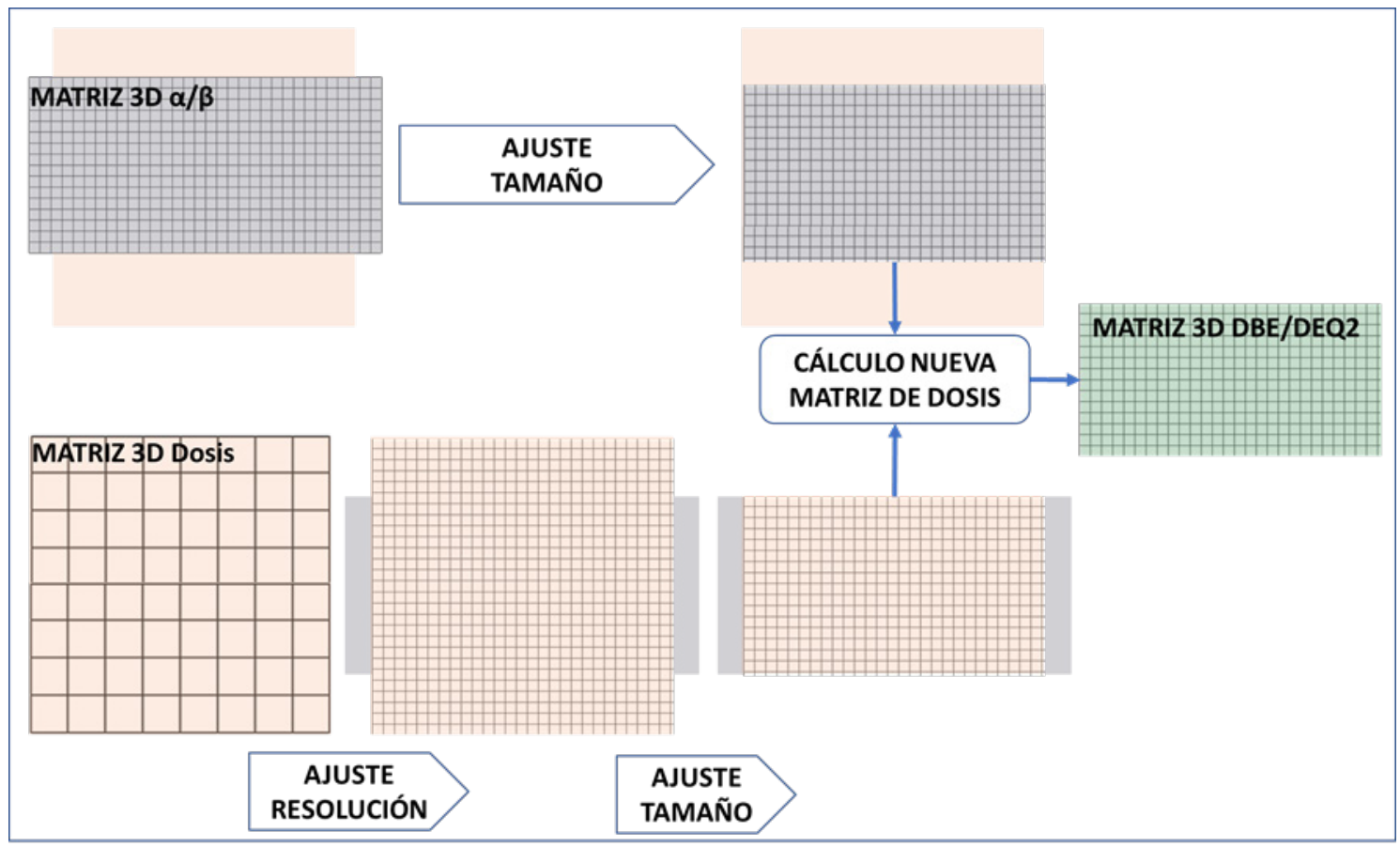

Fig. 2. Flujo de creación de las matrices 3D de dosis modificadas (DBE, DEQ2).

Una vez que ambas matrices tienen la misma resolución, es necesario igualar el tamaño de ambas matrices. Para ello hay que delimitar espacialmente la intersección entre ellas, es decir, delimitar qué volumen del espacio es común, para eliminar aquellas filas y columnas que no lo son e igualar las dimensiones de las dos matrices tal y como se aprecia en la figura 2. Es habitual que haya zonas de la imagen fuera de la malla de cálculo y viceversa. Una vez igualadas la resolución y las dimensiones, obtenemos dos matrices, donde cada elemento de la matriz de dosis $(i, j$, $k$ ) tiene asignado su elemento correspondiente en la matriz $\alpha / \beta(i, j, k)$. Calculamos una nueva matriz de dosis 3D a partir del número de fracciones del plan, la dosis física y el cociente $\alpha / \beta$ correspondiente a cada vóxel. Los elementos de la matriz de dosis 3D tienen una profundidad de 16 bits, por lo que es necesario convertir la nueva matriz de dosis a esta profundidad y obtener un nuevo factor de escalado Dose Grid Scaling. Sobrescribiendo en el archivo original la nueva matriz de dosis 3D y el factor de escalado, generamos un nuevo archivo RT-Dose, importable en el sistema de planificación.

Para realizar una evaluación completa del código, se ha empleado un maniquí homogéneo, formado por diez planchas de metacrilato de $30 \mathrm{~cm} \times 30 \mathrm{~cm}$ apiladas. El estudio de imagen tiene un tamaño de vóxel de 0.797 $\mathrm{mm} \times 0.797 \mathrm{~mm} \times 1.00 \mathrm{~mm}$ y ha sido adquirido para las cuatro posibles orientaciones del paciente, esto es, supino pies primero (SPP), prono pies primero (PPP), supino cabeza primero (SCP) y prono cabeza primero
(PCP). Sobre este maniquí se han contorneado siete diferentes volúmenes de forma cilíndrica $(r=2 \mathrm{~cm}, h=$ $2 \mathrm{~cm}$ ) y con la altura alineada a lo largo de la dirección cráneo-caudal. A cada estructura se le ha asignado un cociente $\alpha / \beta$. Además se ha forzado la densidad de todo el volumen al valor de densidad agua para garantizar la homogeneidad del maniquí. Las estructuras se han colocado de forma espaciada y repartida (fig. 3A). Estas estructuras nos servirán para realizar los cálculos de dosis a punto y a un volumen.

La planificación se ha realizado para un tratamiento de 40 Gy en 10 fracciones, irradiando estas estructuras con la dosis de prescripción y buscando la mayor homogeneidad de la dosis posible. La dosimetría se ha realizado con doce haces de intensidad modulada (IMRT) y una malla de cálculo de resolución $3 \mathrm{~mm} \times$ $3 \mathrm{~mm} \times 3 \mathrm{~mm}$. En el centro de cada volumen se ha colocado un punto de interés (POI) para evaluar la dosis a punto.

\section{Resultados}

Se ha empleado el maniquí anteriormente descrito para evaluar la transformación de la matriz de dosis, para ello es necesario evaluar la correcta identificación de la estructura según su nombre, la asignación por tanto del cociente $\alpha / \beta$ correspondiente y el cálculo final de la DBE y la DEQ2 a un punto. Además, es preciso hacerlo para las distintas orientaciones ya que las estructuras vienen identificadas en el sistema de 

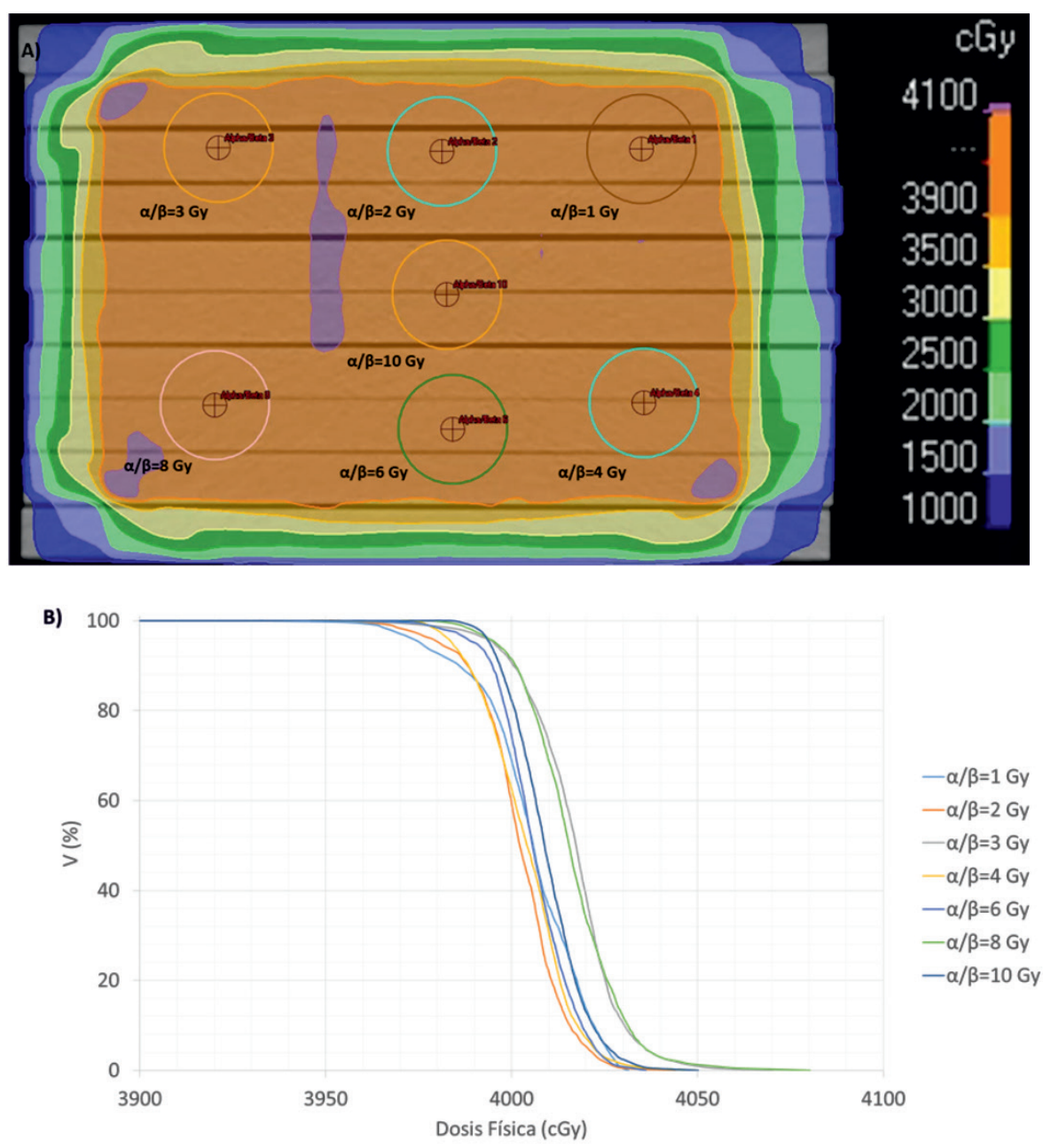

Fig. 3. Plan de tratamiento en dosis física. A) Distribución de dosis en plano axial con puntos de evaluación de dosis por cada ROI con distinto cociente $\alpha / \beta$. B) Detalle del HDV.

coordenadas del paciente, por lo que es necesario validar que el sistema funciona en cada caso. Por último, es importante resaltar que la malla de cálculo puede (y suele) tener un tamaño distinto al estudio de imagen, y por tanto es crítico evaluar la correcta alineación entre la denominada matriz $\alpha / \beta$ y la matriz de dosis. En la figura 4 se muestran las distribuciones de dosis física (a), dosis transformada a DEQ2 (b) y dosis transformada DBE (c). Se puede apreciar el correcto alineado entre la malla de cálculo y las estructuras, mostrando un claro escalón en la dosis, que coincide con los bordes de las estructuras con $\alpha / \beta$ distinto del elegido para el maniquí ( $\alpha / \beta=3 \mathrm{~Gy})$. La Figura 5 muestra los valores de DEQ2 y DBE obtenidos y la dosis física original, superpuestos al valor del cociente $\alpha / \beta$.

Los cálculos de dosis a punto se han realizado para cada posible orientación del paciente en el centro geométrico de cada uno de los siete cilindros donde se ha colocado un punto de interés (POI) donde evaluaremos la dosis. Es importante apuntar que el valor de la dosis absorbida cambia según la orientación ya que son cuatro planificaciones distintas. Los resultados se muestran en la tabla 1.

Cada estructura ha sido etiquetada con un valor distinto del cociente $\alpha / \beta$. Se muestran a continuación las curvas del histograma dosis volumen (HDV) para el tratamiento en términos de DBE (Fig. 6a) y DEQ2 (Fig. 6b).

\section{Discusión}

Los resultados para la transformación de valores de dosis puntual de la tabla 1 muestran una conversión correcta, con la excepción de mínimas desviaciones de unos pocos cGy. Este cálculo, que a priori debería ser exacto, tiene sin embargo tres fuentes de incertidumbre. La primera es la incertidumbre que se introduce al aumentar la resolución de la matriz 3D de dosis ya que implica un proceso de interpolación. La segunda es que el alineamiento de ambas matrices requiere de un desplazamiento, de una matriz sobre la otra, que 

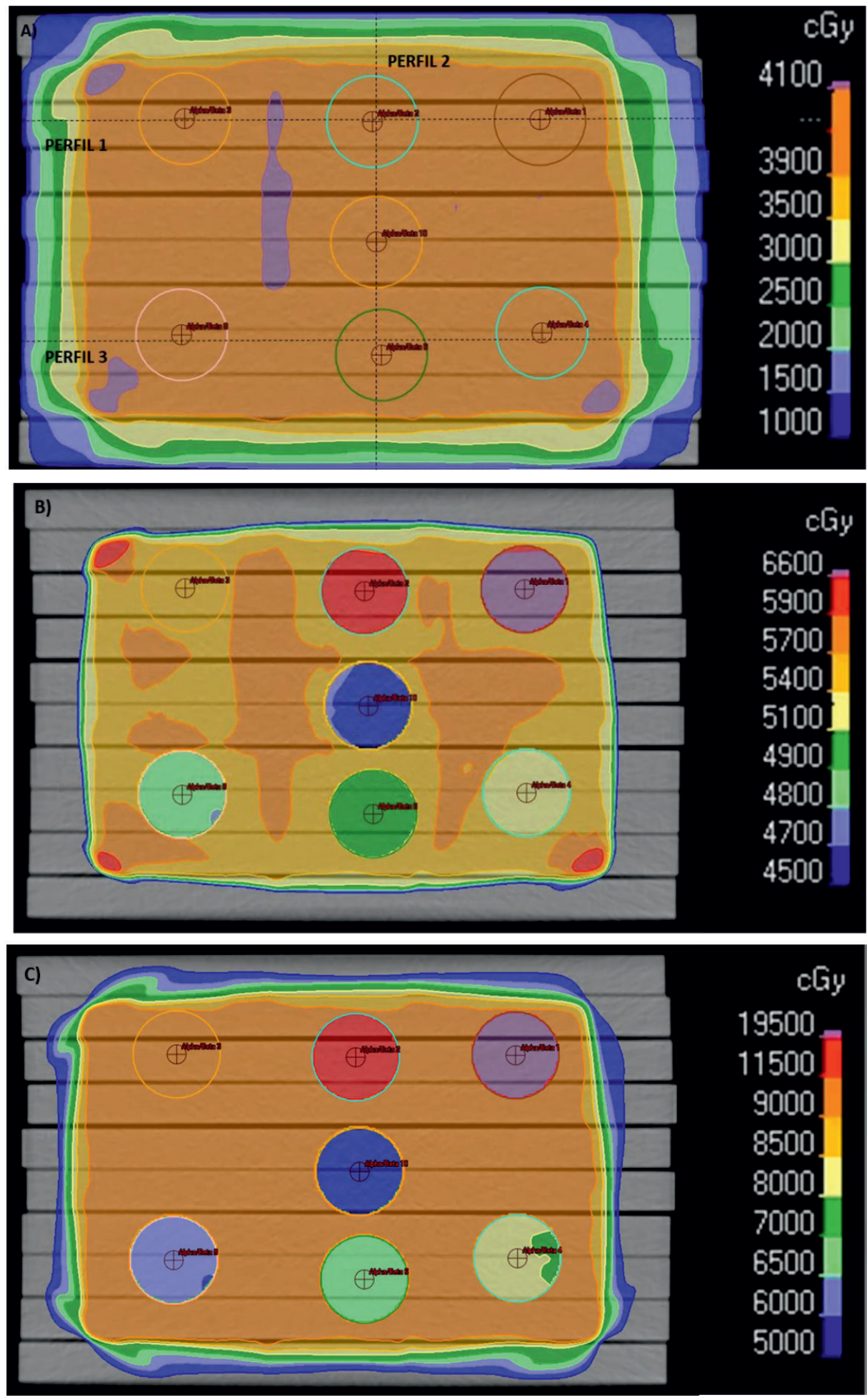

Fig. 4. Distribuciones dosimétricas. A) Dosis física original con los perfiles estudiados. B) DEQ2 C) DBE.

permita alinear perfectamente la posición de ambas. Este desplazamiento siempre es inferior al tamaño de pixel (en cada dimensión) y es dependiente de la relación de tamaños entre el vóxel de imagen y el de dosis. En caso de no ser un número entero existirá un desplazamiento, necesario para el alineamiento de ambas matrices. La tercera es el hecho de que la dosis está almacenada en un entero de 16 bit y por lo tanto, al cambiar la escala asignada al plan, cambia la relación entre el valor de pixel y el de dosis.

El programa asigna adecuadamente el valor del cociente $\alpha / \beta$ según se haya etiquetado la estructura, 
A)
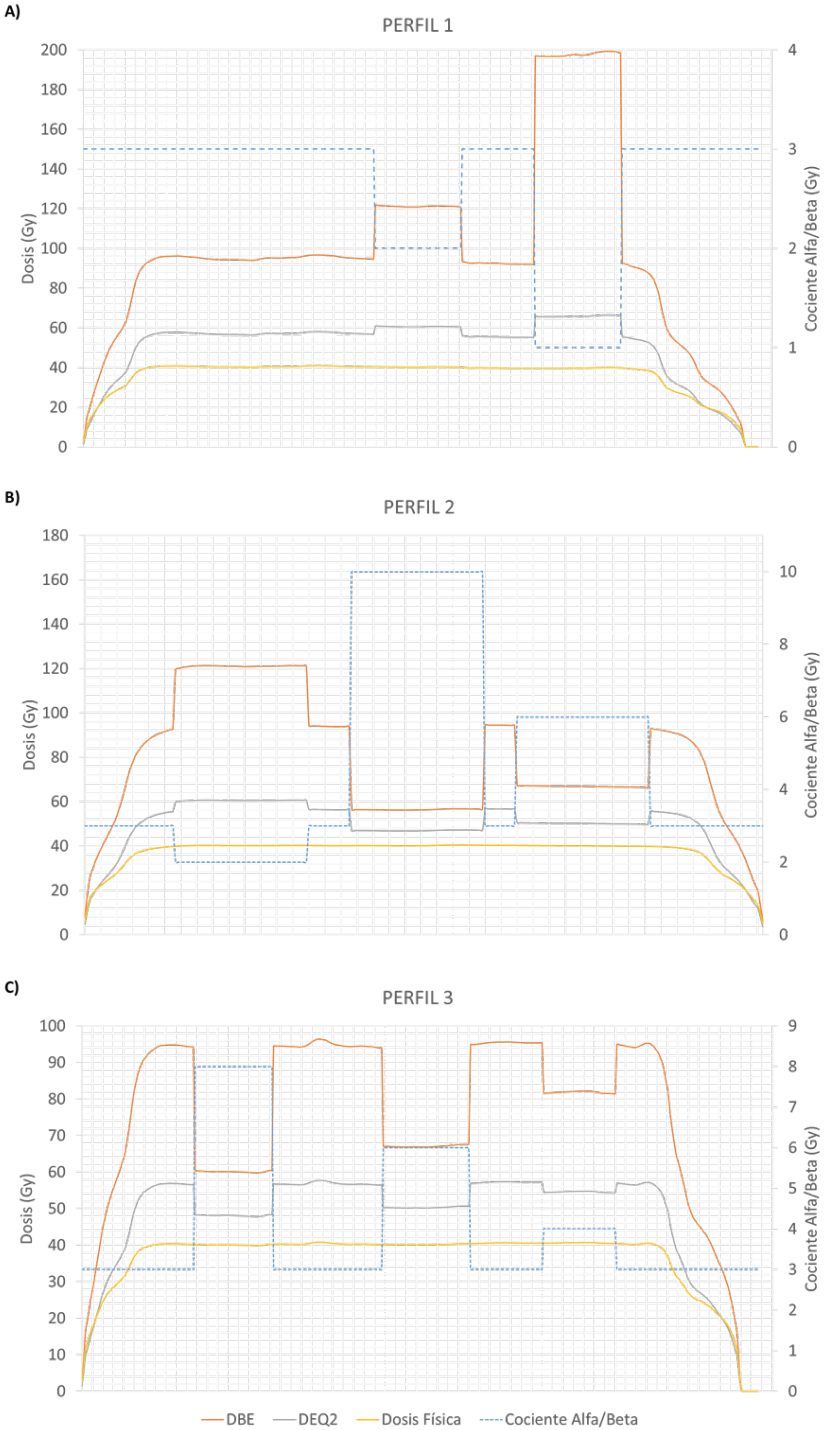

Fig. 5. Perfiles de dosis frente al valor del cociente Alfa/ Beta. Perfiles marcados en la figura 4. A) Perfil 1. B) Perfil 2. C) Perfil 3.

realizando además un ajuste correcto entre el estudio de imagen y la malla de cálculo. La correcta alineación entre las matrices 3D de dosis y de cocientes $\alpha / \beta$ se puede observar en la dosimetría transformada (fig. $4 \mathrm{~b}$ y 4c) y los perfiles de la figura 5 , donde los cambios abruptos de DBE y DEQ2 coinciden con los cambios en el coeficiente $\alpha / \beta$. La Tabla 1 muestra una correcta transformación de las dosis a punto. Esto demuestra que la orientación del paciente no ha influido en el cálculo y por lo tanto la reorientación de matrices 3D se ha realizado adecuadamente.

En el caso de los HDV observamos, tanto para la DBE como para la DEQ2, la aparición de escalones que no se corresponden con la distribución encontrada en el HDV del tratamiento (dosis absorbida). En la figura 6 observamos que todas las estructuras muestran una
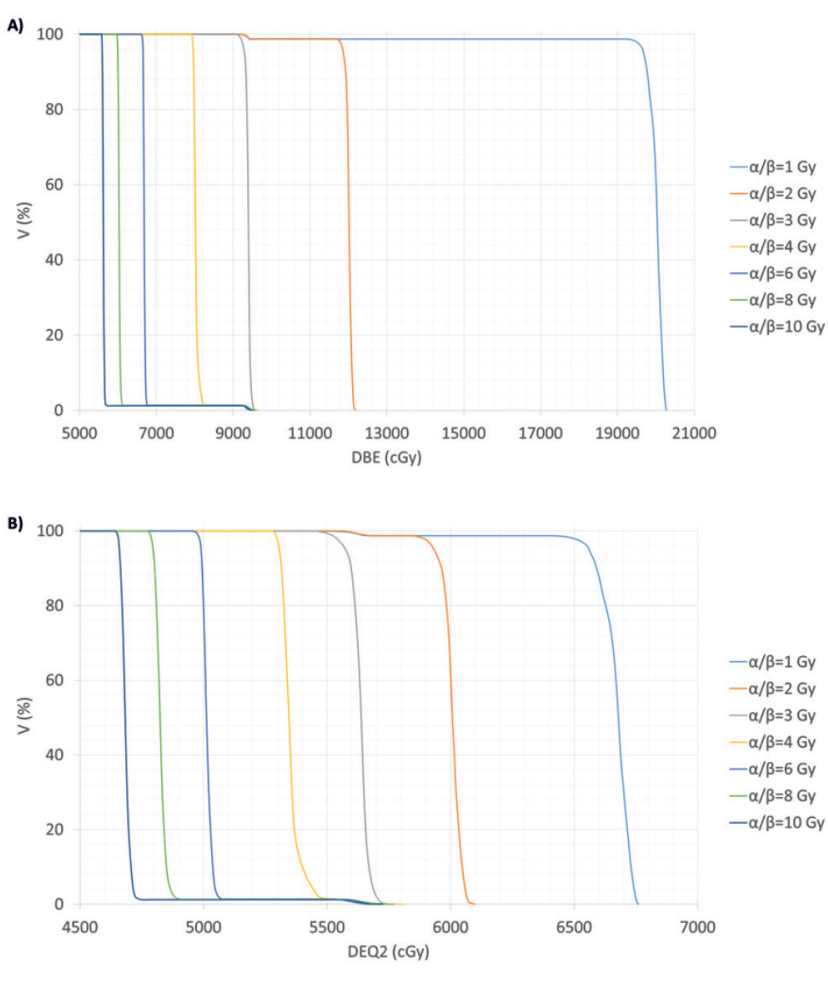

Fig. 6. Histogramas dosis volumen. A) DBE B) DEQ2.

parte del volumen con la DBE y la DEQ2 correspondiente a un valor $\alpha / \beta=3$ Gy. En el caso de las estructuras con $\alpha / \beta<3$ Gy el HDV muestra valores de dosis mínima correspondientes al $\alpha / \beta=3$ Gy. Para $\alpha / \beta>$ 3 Gy las dosis máximas de la estructura corresponden a un $\alpha / \beta=3$ Gy como se aprecia en la figura 7 .

La distribución de valores del cociente $\alpha / \beta$ a lo largo del volumen irradiado no es una función continua, sino escalonada, por lo que vóxeles contiguos pueden tener valores muy diferentes. En consecuencia la distribución de dosis no será continua y presentará escalones. Cada vóxel tiene asociado un valor del cociente $\alpha / \beta$ en función de la estructura a la que pertenezca. Para aquellos que son intersectados por los límites de alguna estructura, como el caso mostrado en la figura 8, es necesario adoptar un criterio. La función que determina si un vóxel queda dentro o fuera de una estructura es la predefinida en Matlab y denominada poly2mask, cuyo método está descrito ampliamente en la documentación. ${ }^{25}$ El cálculo realizado por SPT para el HDV también se ve afectado en el caso de los vóxeles intersectados por los límites de una estructura. El método seguido por el SPT consiste en calcular la fracción de vóxel contenida dentro de la estructura y asignar ese valor de dosis a la fracción de volumen correspondiente como se describe en el manual de usuario. ${ }^{26}$ Es por esto que vóxeles con sólo una fracción de área dentro de una estructura son considerados por Matlab como no contenidos en esta y por tanto obtendrán el cociente $\alpha / \beta$ correspondiente al área externa, como se 
Tabla 1. Resultados del procedimiento de cálculo de DBE y DEQ2 sobre el maniquí para cada una de las estructuras cilíndricas con diferente $\alpha / \beta$ y para las cuatro posibles orientaciones del paciente. Valor en cada punto de evaluación de dosis.

\begin{tabular}{c|c|c|c|c|c|c|c|c}
\hline Posición & POI $\alpha$ I $\beta$ (Gy) & 1 & 2 & 3 & 4 & 6 & 8 & 10 \\
\hline \multirow{4}{*}{ SCP } & Dosis absorbida (cGy) & 4015 & 4004 & 4014 & 4010 & 4003 & 4021 & 4009 \\
\cline { 2 - 9 } & DBE (cGy) & 20125 & 11988 & 9389 & 8033 & 6673 & 6042 & 5592 \\
\cline { 2 - 9 } & DEQ2 (cGy) & 6798 & 5994 & 5633 & 5355 & 5005 & 4833 & 4660 \\
\hline \multirow{4}{*}{ SPP } & Dosis absorbida (cGy) & 4001 & 3994 & 4016 & 3999 & 4002 & 4007 & 4006 \\
\cline { 2 - 9 } & DBE (cGy) & 19956 & 11962 & 9403 & 8026 & 6665 & 6010 & 5617 \\
\cline { 2 - 9 } & DEQ2 (cGy) & 6652 & 5981 & 5642 & 5350 & 4999 & 4808 & 4681 \\
\hline \multirow{4}{*}{ PCP } & Dosis absorbida (cGy) & 4014 & 3997 & 4016 & 4024 & 4003 & 4018 & 4016 \\
\cline { 2 - 9 } & DBE (cGy) & 20083 & 12005 & 9412 & 8057 & 6669 & 6035 & 5630 \\
\cline { 2 - 9 } & DEQ2 (cGy) & 6694 & 6003 & 5647 & 5371 & 5002 & 4829 & 4692 \\
\hline \multirow{3}{*}{ PPP } & Dosis absorbida (cGy) & 4001 & 3994 & 4016 & 3999 & 4002 & 4010 & 4006 \\
\cline { 2 - 9 } & DBE (cGy) & 19956 & 11962 & 9403 & 8026 & 6665 & 6023 & 5617 \\
\cline { 2 - 9 } & DEQ2 (cGy) & 6694 & 6006 & 5616 & 5376 & 4996 & 4722 & 4689
\end{tabular}

aprecia en algunos píxeles de la figura 8. Sin embargo, la fracción de vóxel contenida en la estructura será contabilizada por el SPT para el cálculo del HDV, apareciendo por tanto los escalones que se aprecian en las figuras 7a y 7b, ya que existen vóxeles dentro de la estructura con una dosis calculada a partir de un cociente $\alpha / \beta$ distinto al resto. Esta diferencia es la causa de los escalones apreciados en las curvas de HDV. En el tratamiento valorado, el tejido circundante a las estructuras siempre tiene un cociente $\alpha / \beta$ de valor 3 Gy y por tanto siempre hay una fracción del volumen, de cada estructura considerada, que coincide con la DBE o DEQ2 calculada para este cociente $\alpha / \beta$.
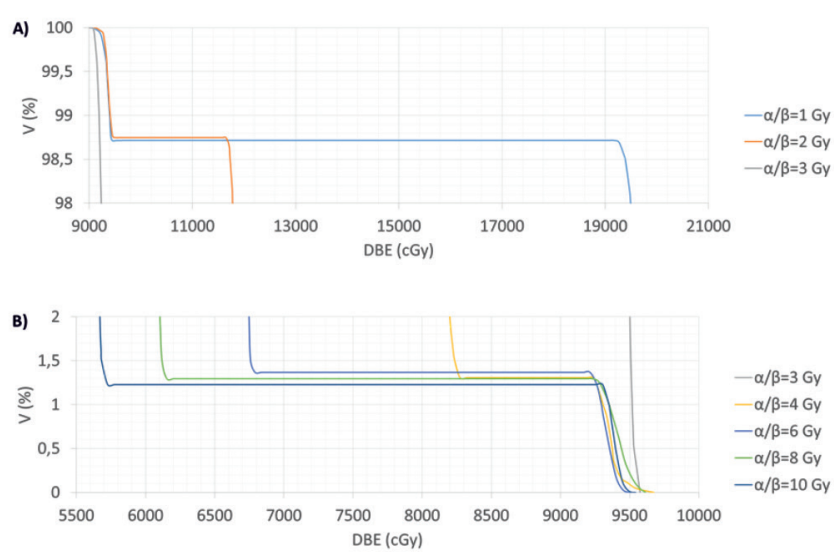

Fig. 7. Detalle efectos de borde en A) Estructuras $\operatorname{con} \alpha / \beta<$ 3 Gy. B) Estructuras con $\alpha / \beta>3$ Gy.

\section{Conclusiones}

Este programa permite un cálculo rápido y sencillo de la DBE y la DEQ2, siendo una herramienta muy útil especialmente en casos complejos que requieren múltiples irradiaciones o re-irradiaciones a fraccionamientos distintos. Ambos casos son cada vez más habituales en los servicios de Oncología Radioterápica. Sin embargo, su uso requiere de una comprensión de su

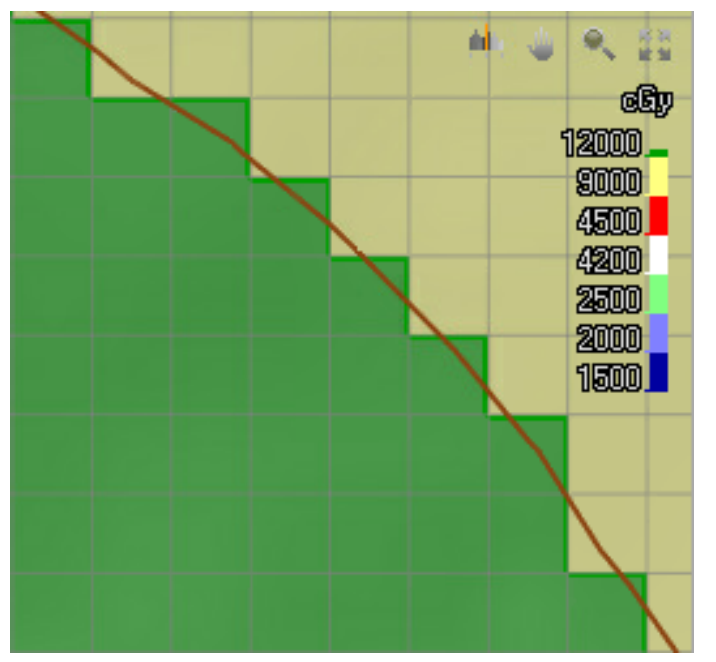

Fig. 8. Frontera entre dos estructuras con distinto $\alpha / \beta$. Se aprecia la asignación del cociente $\alpha / \beta$ en función del valor de dosis mostrado. 
funcionamiento. Las dosis en los márgenes de órganos de riesgo llevan asociadas una incertidumbre dependiente de las estructuras de alrededor. La evaluación de parámetros dosimétricos, especialmente valores como la dosis máxima y en general las dosis a punto, debe realizarse cuidadosamente para evitar errores importantes, especialmente en las zonas limítrofes entre estructuras con diferentes valores del cociente $\alpha / \beta$. Resulta por tanto más apropiada una evaluación a dosis-volumen, utilizando parámetros como la dosis de cobertura al 98\% (D98) o la dosis máxima al 2\% de volumen (D2).

El uso del modelo $L Q$ para esquemas de fraccionamiento que imparten dosis superiores a los 6 - 7 Gy por fracción genera controversia. ${ }^{13,27,28}$ Sin embargo ha demostrado ser el modelo que mejor predicciones hace en el rango entre 1.8 y 20 Gy por fracción ${ }^{29}$ e incluso ha sido empleado en el cálculo de límites de dosis a tejido sano en SBRT a partir de la experiencia tanto en tratamientos con hipofraccionamiento moderado como con fraccionamiento convencional. ${ }^{30}$ Sin embargo el uso de estas matrices de DBE y DEQ2 debe suponer, especialmente en estos casos, una herramienta orientativa que debe ser cuidadosamente evaluada.

Es interesante recordar que la recuperación del tejido sano tras una irradiación es un tema que sigue siendo objeto de investigación actualmente. ${ }^{31-34}$ Futuras versiones podrían incluir modelos de recuperación que permitan un cálculo más realista de las dosis acumuladas.

El código Matlab está disponible para su descarga en la plataforma Github.

\section{Referencias}

1. Bryant AK, Banegas MP, Martinez ME, Mell LK, Murphy JD. Trends in Radiation Therapy among Cancer Survivors in the United States, 2000-2030. Cancer Epidemiol Biomarkers Prev. 2017 Jun;26(6):963-70. doi: 10.1158/1055-9965. EPI-16-1023. Epub 2017 Jan 17. PMID: 28096199.

2. Paradis KC, Mayo C, Owen D, et al. The Special Medical Physics Consult Process for Re-irradiation Patients. Advances in Radiation Oncology. 2019 Oct-Dec;4(4):559-65.

3. Gogineni E, Zhang I, Rana Z, Marrero M, Gill G, Sharma A, Riegel AC, Teckie S, Ghaly M. Quality of Life Outcomes Following Organ-Sparing SBRT in Previously Irradiated Recurrent Head and Neck Cancer. Front Oncol. 2019 Sep 10;9:836.

4. Dionisi F et al. Organs at risk's tolerance and dose limits for head and neck cancer re-irradiation: A literature review. Oral Oncology. Volume 98, November 2019, pp.

5. Scorsetti M, Comito T, Clerici E, et al. Phase II trial on SBRT for unresectable liver metastases: long-term outcome and prognostic factors of survival after 5 years of follow-up. Radiat Oncol. 2018;13(1):234. Published 2018 Nov 26.

6. Bergamo Am, Kauweloa K, Gan G, Shi Z, Daniels K, Crownover R, Narayanasamy G, Stathakis S, Mavroidis P,
Papanikolaou N, Gutierrez A. Correlation between biological effective dose and radiation-induced liver disease from hypofractionated radiotherapy. J Med Phys 2019;44:185-90.

7. Gebhardt BJ, Vargo JA, Ling D, et al. Carotid Dosimetry and the Risk of Carotid Blowout Syndrome After Re-irradiation With Head and Neck Stereotactic Body Radiation Therapy. Int J Radiat Oncol Biol Phys. 2018;101(1):195-200.

8. McDonald MW, Moore MG, Johnstone PA. Risk of carotid blowout after re-irradiation of the head and neck: a systematic review. Int J Radiat Oncol Biol Phys. 2012;82(3):1083-9.

9. Yazici G, Sanlı TY, Cengiz M, et al. A simple strategy to decrease fatal carotid blowout syndrome after stereotactic body reirradiaton for recurrent head and neck cancers. Radiat Oncol. 2013;8:242. Published 2013 Oct 18.

10. Abusaris $H$, Hoogeman $M$, Nuyttens JJ. Re-irradiation: outcome, cumulative dose and toxicity in patients retreated with stereotactic radiotherapy in the abdominal or pelvic region. Technol Cancer Res Treat. 2012;11(6):591-7. doi:10.7785/tcrt.2012.500261

11. Kauweloa KI, Bergamo A, Gutierrez AN, Stathakis S, Papanikolaou N, Mavroidis P. Use of 3D biological effective dose (BED) for optimizing multi-target liver cancer treatments. Australas Phys Eng Sci Med. 2019;42(3):711-8.

12. Fowler JF. The linear-quadratic formula and progress in fractionated radiotherapy. $\mathrm{Br} J$ Radiol. 1989;62(740):679-94. doi:10.1259/0007-1285-62-740-679.

13. Maciá I Garau M. Radiobiology of stereotactic body radiation therapy (SBRT). Reports of practical oncology and radiotherapy 22(2017):86-95.

14. Hopewell JW, Millar WT, Lindquist C, Nordström H, Lidberg P, Gårding J. Application of the concept of biologically effective dose (BED) to patients with Vestibular Schwannomas treated by radiosurgery. J Radiosurg SBRT. 2013;2(4):257-71.

15. Fernández Letón P, Baños Capilla C, Bea Gilabert J, Delgado Rodríguez JM, De Blas Piñol R, Martínez Ortega J, Pérez Moreno JM, Simón de Blas R. Recomendaciones de la Sociedad Española de Física Médica (SEFM) sobre implementación y uso clínico de radioterapia estereotáxica extracraneal (SBRT). Rev Fis Med 2017;18(2):77-142.

16. Kuperman VY. Effect of intratumor heterogeneity on BED for hypofractionated dose regimens. Med Phys. 2019 Oct; 46(10):4690-8. doi: 10.1002/mp.13689. Epub 2019 Aug 16. PMID: 31257607.

17. Sun, J., Zhang, T., Wang, J. et al. Biologically effective dose (BED) of stereotactic body radiation therapy (SBRT) was an important factor of therapeutic efficacy in patients with hepatocellular carcinoma ( $\leq 5 \mathrm{~cm}$ ). BMC Cancer 19, 846 (2019). https://doi.org/10.1186/s12885-019-6063-9

18. García LM, Leblanc J, Wilkins D, et al. Fitting the linear quadratic model to detailed data sets for different dose ranges. Phys Med Biol 2006;51(11):2813-23.

19. Barendsen GW. Dose fractionation, dose rate and iso-effect relationships for normal tissue responses. Int J Radiat Oncol Biol Phys 1982;8(11):1981-97.

20. Kirkpatrick JP, Meyer JJ, Marks LB. The linear-quadratic model is inappropriate to model high dose per fraction effects in radiosurgery. Semin Radiat Oncol 2008;18(4):240-3.

21. Marks LB. Extrapolating hypofractionated radiation schemes from radiosurgery data: regarding Hall et al., IJROBP 21:819824; 1991 and Hall and Brenner, IJROBP 25:381-5; 1993. Int J Radiat Oncol Biol Phys 1995;32:274-6. 
22. Guirado D, et al. Radiobiología Clínica. ISBN:8950734.

23. Digital Imaging and Communications in Medicine. DICOM. Octubre 2020. <www.dicomstandard.org>

24. Kehwar TS. Analytical approach to estimate normal tissue complication probability using best fit of normal tissue tolerance doses into the NTCP equation of the linear quadratic model. J Cancer Res Ther. 2005;1:168-79.

25. Matlab Image Processing Toolbox: User's Guide (R2019a). The Mathworks Inc. Natick, MA, 2019.

26. Raystation 4.7 User Manual. RaySearch Laboratories AB. Estocolmo, Suecia, 2014.

27. Kirkpatrick JP, Brenner DJ, Orton CG. The linear-quadratic model is inappropriate to model high dose per fraction effects in radiosurgery. Med Phys. 2009;36:3381-4.

28. David J. Brenner. The linear-quadratic model is an appropriate methodology for determining iso-effective doses at large doses per fraction. Semin Radiat Oncol. 2008 October; 18(4):234-9. doi:10.1016/j.semradonc.2008.04.004

29. Martin Brown J, Carlson David J, Brenner David J. The Tumor Radiobiology of SRS and SBRT: Are More than the 5
R's Involved? Int J Radiat Oncol Biol Phys. 2014 February 1; 88(2): 254-62. doi:10.1016/j.jirobp.2013.07.022

30. Pollom EL, Chin AL, Diehn M, Loo BW, Chang DT. Normal Tissue Constraints for Abdominal and Thoracic Stereotactic Body Radiotherapy. Semin Radiat Oncol. 2017 Jul;27(3):197208. doi: 10.1016/j.semradonc.2017.02.001. Epub 2017 Feb 20. PMID: 28577827.

31. Nieder C, Milas L, Ang KK. Tissue tolerance to reirradiation. Semin Radiat Oncol. 2000 Jul;10(3):200-9. doi: 10.1053/ srao.2000.6593. PMID: 11034631.

32. Armstrong S, Hoskin P. Complex Clinical Decision-Making Process of Re-Irradiation. Clin Oncol (R Coll Radiol). 2020 Nov;32(11):688-703. doi: 10.1016/j.clon.2020.07.023. Epub 2020 Sep 3. PMID: 32893056.

33. Nieder C. Second re-irradiation: A delicate balance between safety and efficacy. Phys Med. 2019 Feb;58:155-8. doi: 10.1016/j.ejmp.2019.01.004. Epub 2019 Jan 8. PMID: 3063 5148.

34. Stewart FA, van der Kogel AJ. Retreatment Tolerance of Normal Tissues. Semin Radiat Oncol. 1994 Apr;4(2):103-11. doi: 10.1053/SRA000400103. PMID: 10717096. 


\section{SIEMENS}

\section{Small cells don't have to lead to big problems.}

Siemens answers help doctors detect diseases earlier, saving costs and extending lives.

When diseases are caught early, they make less of an impact on everyone. That's because a large majority of today's healthcare costs go toward treating the late stages of diseases like cancer and heart disease. With Siemens advanced diagnostic technology, doctors can accurately identify these killers earlier. So patients get the treatment they need sooner. Which helps save lives and cuts costs. Wherever there are tough healthcare questions, we're answering them. 